\title{
ML CFO and PO Estimation in DCT OFDM Systems under Non-Circular Transmissions
}

\author{
Tao Cui \\ Department of Electrical Engineering \\ California Institute of Technology \\ Pasadena, CA 91125, USA \\ Email: taocui@caltech.edu
}

\author{
Feifei Gao and A. Nallanathan \\ Department of Electrical \\ and Computer Engineering \\ National University of Singapore \\ Singapore 119260 \\ Email: $\{$ feifeigao, elena $\} @$ nus.edu.sg
}

\author{
Chintha Tellambura \\ Department of Electrical \\ and Computer Engineering \\ University of Alberta \\ Edmonton, AB T6G 2V4, Canada \\ Email: chintha@ece.ualberta.ca
}

\begin{abstract}
Frequency synchronization is one of the most important components in orthogonal frequency-division multiplexing (OFDM) systems. Recently, the discrete cosine transform (DCT) based OFDM system has received wide attentions due to several advantages. Hence, the study of frequency synchronization issue for this newly raised system is well on its time. To provide a thorough study, we consider the non-circular transmissions, and the results can be easily generated to circular transmissions if the elliptic variance is set to zero. We present three joint maximum likelihood (ML) carrier frequency offset (CFO) and phase offset (PO) estimators. From both the theoretical analysis and the numerical comparisons, we found new advantages of the DCT-OFDM over the traditional discrete Fourier transform (DFT) based OFDM. These advantages, as well as those already studied in the early works on DCT-OFDM, support the belief that the DCT-OFDM is a new promising multi-carrier modulation (MCM) scheme.
\end{abstract}

\section{INTRODUCTION}

The traditional DFT based OFDM has already been enjoying its success when it is applied to European digital audio/video broadcasting (DAB, DVB) [1], [2], high performance local area network (HIPERLAN) [3] and IEEE 802.11a wireless LAN standards [4]. Another promising MCM, known as the DCT based OFDM has been proposed recently in [5], [6], where it is shown that the DCT-OFDM can, some times, offer additional benefits over the DFT-OFDM.

In MCM systems, it is well known that a CFO, caused by oscillators mismatch or Doppler effects, destroys the subcarriers orthogonality, and results in a substantial bit error rate (BER) degradation [7]. In the pioneer work [8], ML CFO estimation was developed for DFT-OFDM systems. The algorithm exploited the existence of the cyclic prefix (CP) and was mainly proposed for additive white Gaussian noise (AWGN) channels. However, this method fails to provide the $\mathrm{PO}$ estimation which is very important when the channel is assumed to be AWGN.

Although circularity is a common hypothesis for narrowband signal analysis, non-circular transmission has also received lots of attentions [9]. In fact, many modulations of practical interest, such as BPSK, M-ASK, PAM, DBPSK, OQPSK, OQAM, MSK, GMSK, contain non-circular process. It is also noted that most popular non-circular signals currently adopted are BPSK, DBPSK, M-ASK, and PAM, all of which are real signals. Since the DCT uses only real arithmetic, as opposed to the complex valued DFT, adopting DCT-OFDM under non-circular transmissions can reduce the signal processing complexity/power consumption and avoids inphase/quadrature (I/Q) imbalance problems. It is thus more important to study the non-circular transmissions for the DCTOFDM system.

In this paper, we derive three joint $\mathrm{ML}$ CFO and $\mathrm{PO}$ estimators (MLE) for DCT OFDM, assuming the non-circular transmissions. Similar to [8], all these algorithms are currently proposed for AWGN channel. It is known that the CFO estimation range in DFT OFDM is restricted to one subcarrier spacing no matter whether circular or non-circular signals are transmitted [10]. Nevertheless, due to the anti-symmetry introduced in DCT-OFDM, a full range CFO estimation is achieved. Another advantage by using DCT-OFDM is that it can provide more accurate and more robust estimation than the DFT-OFDM.

This paper is organized as follows. Section II briefly introduces the non-circular transmissions. In Section III, we derive three joint $\mathrm{CFO}$ and PO estimators. Meaningful discussions are provided at the end of this section. Section IV shows the simulation results illustrating the effectiveness of our algorithms. Finally, conclusions are made in Section V.

\section{Non-Circular Multi-Carrier Transmissions}

The $m$ th OFDM block in the frequency domain is represented as

$$
\mathbf{s}_{m}=\left[s_{m, 0}, s_{m, 1}, \ldots, s_{m, N-1}\right]^{T}, \quad m=0,1,2, \ldots,
$$

where $N$ denotes the number of the subcarriers adopted. The complex random variable $s_{m, i}$ is said to be non-circular at the order 2, if the elliptic variance $\mathrm{E}\left\{s_{m, i} s_{m, i}\right\}$ is not zero. Therefore, we assume each $s_{m, i}$ has its variance and elliptic variance as

$$
\begin{aligned}
& \mathrm{E}\left\{s_{m, i_{1}} s_{m, i_{2}}^{*}\right\}=E_{s} \delta_{i_{1} i_{2}}, \\
& \mathrm{E}\left\{s_{m, i_{1}} s_{m, i_{2}}\right\}=E_{c} \delta_{i_{1} i_{2}},
\end{aligned}
$$

where $\delta_{a b}$ is the Kronecker delta function, $E_{s}$ denotes the average signal power, and $E_{c}$ may be a complex number 
with $\left|E_{c}\right| \leq E_{s}$. Here, symbols transmitted on different subcarriers are reasonably assumed independent from each other. However, the symbol on the same subcarrier may be dependent from block to block. Clearly, lots of practical signal modulations contain non-circularity.

Example 1: BPSK modulation. Let $s_{m, i} \in\left\{\sqrt{E_{s}},-\sqrt{E_{s}}\right\}$. Then, we have

$$
E_{s}=\mathrm{E}\left\{s_{m, i} s_{m, i}^{*}\right\}=\mathrm{E}\left\{s_{m, i} s_{m, i}\right\}=E_{c} .
$$

Example 2: MSK modulation. OFDM-MSK modulation has recently attracted lots of interests, whose original form was proposed in [11]. In this new modulation scheme, different branches of MSK symbols are transmitted through different subcarriers. The MSK symbol on the $i$ th subcarrier is

$$
s_{m, i}=\overline{E_{s}} \exp j \pi h \quad a_{m, i}+{ }_{q=0}^{m-1} a_{q, i} \quad,
$$

where $a_{m, i} \in\{ \pm 1\}$ is independently generated and $h$ is the modulation indices. Obviously,

$$
\mathrm{E}\left\{s_{m, i} s_{m, i}^{*}\right\}=E_{s}
$$

whereas,

$$
\begin{aligned}
E_{c} & =\mathrm{E}\left\{s_{m, i} s_{m, i}\right\}=E_{s} \quad \mathrm{E} e^{\left(j 2 \pi h a_{m, i}\right)} \quad m+1 \\
& =E_{s} \frac{1}{2} e^{j 2 \pi h}+e^{-j 2 \pi h} \stackrel{m+1}{=} E_{s}(\cos (2 \pi h))^{m+1} .
\end{aligned}
$$

When $h=1 / 2$, as usually the case for MSK, $E_{c}=$ $(-1)^{m+1} E_{s}$. Clearly, OFDM-MSK also includes noncircularity.

\section{Discrete Cosine Transform Based OFDM SYSTEM}

\section{A. System Model}

In a DCT OFDM system, instead of performing the inverse DFT (IDFT), we apply inverse DCT (IDCT) to the $m$ th time domain OFDM block, resulting in

$$
\mathbf{u}_{m}=\left[u_{m, 0}, u_{m, 1}, \ldots, u_{m, N-1}\right]^{T}=\mathbf{D}^{T} \mathbf{s}_{m},
$$

where $\mathbf{D}$ is the DCT matrix with the $(a, b)$ th entry

$$
[\mathbf{D}]_{a b}=\beta_{a} \quad \frac{\overline{2}}{N} \cos \frac{\pi(a-1)(2 b-1)}{2 N}, \quad a, b=1, \ldots, N .
$$

and

$$
\beta_{a}=\begin{array}{ll}
1 / \sqrt{2}, & a=1 \\
1, & \text { otherwise }
\end{array} .
$$

If the number of the subcarriers is sufficiently large, $u_{m, i}, i=$ $0, \ldots, N-1$ can be well approximated as having a Gaussian distribution [12]. It can be readily verified that,

$$
\begin{aligned}
& \mathrm{E}\left\{\mathbf{u}_{m} \mathbf{u}_{m}^{H}\right\}=\operatorname{DE}\left\{\mathbf{s}_{m} \mathbf{s}_{m}^{H}\right\} \mathbf{D}^{H}=E_{s} \mathbf{I}_{N}, \\
& \mathrm{E}\left\{\mathbf{u}_{m} \mathbf{u}_{m}^{T}\right\}=\operatorname{DE}\left\{\mathbf{s}_{m} \mathbf{s}_{m}^{T}\right\} \mathbf{D}^{T}=E_{c} \mathbf{I}_{N} .
\end{aligned}
$$

Note that, $u_{m, i}$, the time domain symbol after the IDCT, are independent from each other.
Suppose that the maximum channel delay is no longer than $L T_{s}$, where $T_{s}$ is the sampling period. From [5], we know that in order to diagonalize the channel matrix for the DCTOFDM, we need to insert both the prefix and the suffix of length $\mu=\frac{L}{2}$ into $\mathbf{u}_{m}$. Therefore, the effective OFDM block length is $(N+2 \mu) T_{s}$ and the symbols transmitted are represented by

$$
\mathbf{x}_{m}=\left[x_{m, 0}, x_{m, 1}, \ldots, x_{m, N+2 \mu-1}\right]^{T}=\mathbf{T}_{\mathrm{ps}} \mathbf{u}_{m},
$$

where

$$
\mathbf{T}_{\mathrm{ps}}=\begin{array}{cc}
\mathbf{V}_{\mu} & \mathbf{0}_{\mu \times(N-\mu)} \\
\mathbf{I}_{N} \\
\mathbf{0}_{\mu \times(N-\mu)} & \mathbf{V}_{\mu}
\end{array},
$$

and $\mathbf{V}_{\mu}$ is the $\mu \times \mu$ anti-identity matrix:

$$
\mathbf{V}_{\mu}=\begin{array}{ccc}
0 & \ldots & 1 \\
\vdots & . & \vdots \\
1 & \ldots & 0
\end{array} .
$$

As in [8], we will only consider the AWGN channel for the time being but still keep both the prefix and the suffix for the estimation purpose. The baseband received signal could be expressed as

$$
\begin{aligned}
\mathbf{r}_{m} & =\left[r_{m, 0}, r_{m, 1}, \ldots, r_{m, N+2 \mu-1}\right]^{T} \\
& =e^{j 2 \pi m(N+2 \mu) \varepsilon_{0}+j \phi_{0}} \boldsymbol{\Gamma}\left(\varepsilon_{0}\right) \mathbf{x}_{m}+\mathbf{n}_{m}
\end{aligned}
$$

where $\varepsilon_{0}=f_{0} T_{s}$ is the normalized CFO by the sampling rate, and $\phi_{0}$ is the PO. Each elements in $\mathbf{n}_{m}$ represents independent AWGN with variance $E_{n}$, and $\boldsymbol{\Gamma}\left(\varepsilon_{0}\right)$ is the diagonal CFO matrix of the form

$$
\boldsymbol{\Gamma}\left(\varepsilon_{0}\right)=\operatorname{diag} \quad 1, e^{j 2 \pi \varepsilon_{0}}, \ldots, e^{j 2 \pi(N+2 \mu-1) \varepsilon_{0}} .
$$

\section{B. The Joint $C F O$ and PO Estimation}

The frequency synchronization should be carried on after the first OFDM block is received. Therefore, we consider the OFDM block with index number $m=0$, and the index $m$ will be dropped for notation simplicity in the remaining of this paper unless otherwise mentioned. Define

$$
\begin{aligned}
& \mathbf{A}=E_{s} \mathbf{T}_{\mathrm{ps}} \mathbf{T}_{\mathrm{ps}}^{T}+E_{n} \mathbf{I}_{N+2 \mu}, \\
& \mathbf{B}=E_{c} \mathbf{T}_{\mathrm{ps}} \mathbf{T}_{\mathrm{ps}}^{T} .
\end{aligned}
$$

Clearly, we have $\mathbf{A}^{*}=\mathbf{A}$ while $\mathbf{B}^{*} \neq \mathbf{B}$ unless $E_{c}$ is a real number. Nevertheless, $\mathbf{B}^{*}$ can be represented as $\mathbf{B}^{H}$. Consequently, the covariance and the elliptic covariance of $\mathbf{r}$ are $\boldsymbol{\Gamma}\left(\varepsilon_{0}\right) \mathbf{A} \boldsymbol{\Gamma}\left(\varepsilon_{0}\right)^{H}$ and $e^{j 2 \phi_{0}} \boldsymbol{\Gamma}\left(\varepsilon_{0}\right) \mathbf{B} \boldsymbol{\Gamma}\left(\varepsilon_{0}\right)$, respectively. Therefore, $\mathbf{r}$ is a non-circular Gaussian random vector and should be characterized by the joint probability density function (PDF) [13]:

$$
\begin{aligned}
& f\left(\mathbf{r}, \mathbf{r}^{*} \mid \varepsilon_{0}, \phi_{0}\right)= \frac{1}{\pi^{N+L}(\operatorname{det}\{\mathbf{R}\})^{1 / 2}} \\
& \times \exp -\frac{1}{2}\left[\mathbf{r}^{H}, \mathbf{r}^{T}\right] \mathbf{R}^{-1} \quad \mathbf{r} \\
& \mathbf{r}^{*}
\end{aligned}
$$




$$
\eta+\frac{2\left(2 E_{s}^{2}+E_{s} E_{n}-2\left|E_{c}\right|^{2}\right)}{E_{n}\left(4 E_{s}^{2}+4 E_{s} E_{n}+E_{n}^{2}-4\left|E_{c}\right|^{2}\right)}{ }_{i=0}^{\mu-1} \Re \quad\left(r_{i}^{*} r_{2 \mu-i-1}+r_{i+N}^{*} r_{N+2 \mu-i-1}\right) e^{j 2 \pi(2 i-2 \mu+1) \varepsilon}
$$

$$
\begin{aligned}
& \frac{2 E_{c}^{*}}{4 E_{s}^{2}+4 E_{s} E_{n}+E_{n}^{2}-4\left|E_{c}\right|^{2}}{ }_{i=0}^{\mu-1} r_{i}^{*} r_{2 \mu-i-1}^{*} e^{-j 2 \pi(2 \mu-1) \varepsilon}+{ }_{i=0}^{2 \mu-1} r_{i+N}^{*} r_{N+2 \mu-i-1}^{*} e^{-j 2 \pi(2 N+2 \mu-1) \varepsilon} \\
& +\frac{E_{c}^{*}}{4 E_{s}^{2}+4 E_{s} E_{n}+E_{n}^{2}-4\left|E_{c}\right|^{2}}{ }_{i=0}^{2 \mu-1} r_{i}^{*} r_{i}^{*} e^{-j 2 \pi(2 i) \varepsilon}+{ }_{i=N}^{N+2 \mu-1} r_{i}^{*} r_{i}^{*} e^{-j 2 \pi(2 i) \varepsilon} \\
& +\frac{E_{c}^{*}}{E_{s}^{2}+2 E_{s} E_{n}+E_{n}^{2}-\left|E_{c}\right|^{2}}{ }_{i=2 \mu}^{N-1} r_{i}^{*} r_{i}^{*} e^{-j 2 \pi(2 i) \varepsilon}
\end{aligned}
$$

where $\mathbf{R}$ is the covariance matrix of $\left[\mathbf{r}^{T}, \mathbf{r}^{H}\right]^{T}$, defined as

$$
\begin{aligned}
& \mathbf{R}=\mathrm{E} \quad \underset{\mathbf{r}^{*}}{\mathbf{r}}\left[\mathbf{r}^{H}, \mathbf{r}^{T}\right]
\end{aligned}
$$

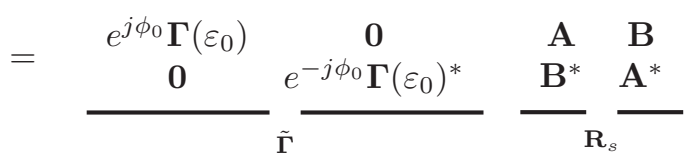

$$
\begin{aligned}
& \begin{array}{cc}
e^{-j \phi_{0}} \boldsymbol{\Gamma}\left(\varepsilon_{0}\right)^{*} & \mathbf{0} \\
\mathbf{0} & e^{j \phi_{0}} \mathbf{\Gamma}\left(\varepsilon_{0}\right)
\end{array} .
\end{aligned}
$$

From the inversion of the partitioned matrix lemma, we obtain

$$
\mathbf{R}_{s}^{-1}=\begin{array}{cc}
\mathbf{P} & -\mathbf{Q} \\
-\mathbf{Q}^{*} & \mathbf{P}^{*}
\end{array}
$$

where

$$
\begin{aligned}
& \mathbf{P}=\left(\mathbf{A}-\mathbf{B}^{H} \mathbf{A}^{-1} \mathbf{B}\right)^{-*}, \\
& \mathbf{Q}=\mathbf{A}^{-1} \mathbf{B P}^{*} .
\end{aligned}
$$

and $(\cdot)^{-*}$ denotes $\left((\cdot)^{*}\right)^{-1}$. Then $\mathbf{R}^{-1}=\tilde{\boldsymbol{\Gamma}} \mathbf{R}_{s}^{-1} \tilde{\boldsymbol{\Gamma}}^{H}$. From (21), we know that

$$
\operatorname{det}\{\mathbf{R}\}=\operatorname{det}\{\tilde{\boldsymbol{\Gamma}}\} \operatorname{det}\left\{\mathbf{R}_{s}\right\} \operatorname{det}\left\{\tilde{\boldsymbol{\Gamma}}^{H}\right\}=\operatorname{det}\left\{\mathbf{R}_{s}\right\} \text {. }
$$

Therefore, $\operatorname{det}\{\mathbf{R}\}$ is only a constant, and it is sufficient to consider the log-likelihood function only. Hereafter, we will provide three different types of the ML joint CFO and PO estimators.

1) MLE1: When $\varepsilon_{0}, \phi_{0}$ are both considered as deterministic values, their ML estimates can be found from

$$
\begin{aligned}
\left\{\hat{\varepsilon}_{0}, \hat{\phi}_{0}\right\} & =\arg \max _{\varepsilon, \phi} \log f\left(\mathbf{r}, \mathbf{r}^{*} \mid \varepsilon, \phi\right) \\
& =\arg \max _{\varepsilon, \phi}-\left[\mathbf{r}^{H}, \mathbf{r}^{T}\right] \mathbf{R}^{-1} \underset{\mathbf{r}^{*}}{\mathbf{r}},
\end{aligned}
$$

where $\varepsilon, \phi$ are the trial parameters. After some manipulations, the ML estimates $\hat{\varepsilon}_{0}$ and $\hat{\phi}_{0}$ can be obtained by

$$
\left\{\hat{\varepsilon}_{0}, \hat{\phi}_{0}\right\}=\arg \max _{\varepsilon, \phi}-\mathbf{r}^{H} \mathbf{Y r}+\Re\left\{e^{-j 2 \phi} \mathbf{r}^{T} \mathbf{U r}\right\},
$$

where

$$
\begin{aligned}
\mathbf{Y} & =\boldsymbol{\Gamma}(\varepsilon) \mathbf{P} \boldsymbol{\Gamma}(\varepsilon)^{*} \\
\mathbf{U} & =\boldsymbol{\Gamma}(\varepsilon)^{*} \mathbf{Q}^{*} \boldsymbol{\Gamma}(\varepsilon)^{*}
\end{aligned}
$$

Due to the special structure of $\mathbf{A}$ and $\mathbf{B}$, when $2 \mu<N$, $-\mathbf{r}^{H} \mathbf{Y r}$ can be rewritten as $(30)^{1}$, where $\eta$ is some unimportant parameters unspecified for the concise of the paper, and $\mathbf{r}^{T} \mathbf{U r}$ can be expressed as in (31). Note that only the second term in (27) is related with $\phi$. Then PO can be estimated via

$$
\hat{\phi}_{0}=\arg \max _{\phi} \Re\left\{e^{-j 2 \phi} \mathbf{r}^{T} \mathbf{U} \mathbf{r}\right\}=\frac{1}{2} \angle \mathbf{r}^{T} \mathbf{U r},
$$

where $\angle$ is used for extracting the angle. Clearly, the estimation range of PO is $[-\pi / 2, \pi / 2)$. Substituting (32) back into (27), $\hat{\varepsilon}_{0}$ can be found from

$$
\hat{\varepsilon}_{0}=\arg \max _{\varepsilon} \frac{-\mathbf{r}^{H} \mathbf{Y r}}{g(\varepsilon)}+\frac{\left|\mathbf{r}^{T} \mathbf{U r}\right|}{} .
$$

It is noted that the noise power $E_{n}$ is required by the estimator (27). This is not unexpected as is seen from [8], where the noise power is used to estimate the TO. The study on the noise power estimation can be found from [14] and references therein.

2) MLE2: Strictly speaking, MLE1 is not an ML estimator but a generalized likelihood ratio testing estimator [15]. To realize the true $\mathrm{ML}$ detection, we have to pursue a two dimensional search for both $\varepsilon$ and $\phi$ from equation (27). Due to its high complexity by performing two dimensional search, the MLE2 is only of theoretical interest and is proposed here as a benchmark for comparison with other estimators.

3) MLE3: When the statistics of either $\phi_{0}$ or $\varepsilon_{0}$ is known, the ML estimation can be modified by using the marginal likelihood function. It is natural to assume that $\phi_{0}$ is a uniformly distributed random variable over $[-\pi, \pi)$. From (32), we know that $f\left(\mathbf{r}, \mathbf{r}^{*} \mid \varepsilon, \phi\right)$ is a periodic function of $\phi$ with the period $\pi$. Therefore we will assume that $\phi_{0}$ is uniformly distributed over the region $[-\pi / 2, \pi / 2)^{2}$. The average of $f\left(\mathbf{r}, \mathbf{r}^{*} \mid \varepsilon, \phi\right)$ over $\phi$ gives the marginal likelihood

\footnotetext{
${ }^{1}$ Since in practice, $2 \mu<N$ is always satisfied, we will only provide the explicit expression of the estimators for this case.

${ }^{2}$ Normally the PO should be uniformly distributed over the region $[-\pi, \pi)$. However, if $\left|\phi_{0}\right|>\pi / 2$, then only $\left(\phi_{0} \bmod \pi\right)-\pi / 2$ can be found by the proposed estimator. Therefore the effective PO can reasonably be assumed to be uniformly distributed over the region $[-\pi / 2, \pi / 2)$.
} 
function $f\left(\mathbf{r}, \mathbf{r}^{*} \mid \varepsilon\right)$, which removes the likelihood dependence on $\phi$. This marginal likelihood function can be expressed as

$$
\begin{aligned}
f\left(\mathbf{r}, \mathbf{r}^{*} \mid \varepsilon\right) & =\mathrm{E}_{\phi}\left\{f\left(\mathbf{r}, \mathbf{r}^{*} \mid \varepsilon, \phi\right)\right\} \\
& =K \exp -\mathbf{r}^{H} \mathbf{Y r} \quad \mathrm{E}_{\phi} \exp \left(\Re\left\{e^{-j 2 \phi} \mathbf{r}^{T} \mathbf{U} \mathbf{r}\right\}\right) \\
& =\frac{K}{\pi} \exp -\mathbf{r}^{H} \mathbf{Y r} \quad \underset{-\pi / 2}{\exp } \Re\left\{e^{-j 2 \phi} \mathbf{r}^{T} \mathbf{U r}\right\} \quad d \phi,
\end{aligned}
$$

where

$$
K=\frac{1}{\pi^{N+L}(\operatorname{det}\{\mathbf{R}\})^{1 / 2}} .
$$

For notational simplicity, we define $w=\left|\mathbf{r}^{T} \mathbf{U r}\right|$ and $\psi=$ $\arg \mathbf{r}^{T} \mathbf{U r}$. Then

$$
\begin{array}{cccc} 
& \quad \exp _{-\pi / 2}^{\pi / 2} \Re\left\{e^{-j 2 \phi} \mathbf{r}^{T} \mathbf{U r}\right\} & d \phi= & \underset{-\pi / 2}{\pi / 2} \exp (w \cos (\psi-2 \phi)) d \phi \\
=\frac{1}{2} & \underset{-\pi-\psi}{\pi / 2}(w \cos t) d t={ }_{0}^{\pi} \exp (w \cos t) d t=\pi I_{0}(w),(36)
\end{array}
$$

where $I_{0}(w)$ is the zeroth order modified Bessel function of the first kind. Finally, the expression for $f\left(\mathbf{r}, \mathbf{r}^{*} \mid \varepsilon\right)$ is

$$
f\left(\mathbf{r}, \mathbf{r}^{*} \mid \varepsilon\right)=K \exp -\mathbf{r}^{H} \mathbf{Y r} \quad I_{0}\left|\mathbf{r}^{T} \mathbf{U r}\right| .
$$

and $\varepsilon_{0}$ should be estimated from

$$
\begin{aligned}
\hat{\varepsilon}_{0} & =\arg \max _{\varepsilon} f\left(\mathbf{r}, \mathbf{r}^{*} \mid \varepsilon\right) \\
& =\arg \max _{\varepsilon} \exp \quad-\mathbf{r}^{H} \mathbf{Y r} \quad I_{0} \quad\left|\mathbf{r}^{T} \mathbf{U r}\right| .
\end{aligned}
$$

For high SNR, $I_{0}(w)$ can be approximated by

$$
I_{0}(w) \approx \frac{e^{w}}{\sqrt{2 \pi w}}
$$

and $f\left(\mathbf{r}, \mathbf{r}^{*} \mid \varepsilon\right)$ is rewritten as

$f\left(\mathbf{r}, \mathbf{r}^{*} \mid \varepsilon\right) \approx K \frac{\exp -\mathbf{r}^{H} \mathbf{Y r}+\left|\mathbf{r}^{T} \mathbf{U r}\right|}{\sqrt{2 \pi}\left|\mathbf{r}^{T} \mathbf{U r}\right|^{1 / 2}}=K \frac{e^{g(\varepsilon)}}{\sqrt{2 \pi}\left|\mathbf{r}^{T} \mathbf{U} \mathbf{r}\right|^{1 / 2}}$

The ML estimation of $\varepsilon$ can be found from

$$
\hat{\varepsilon}_{0}=\arg \max _{\varepsilon} \frac{e^{g(\varepsilon)}}{\left|\mathbf{r}^{T} \mathbf{U r}\right|^{1 / 2}} .
$$

Unfortunately, a reasonable assumption on the distribution of $\varepsilon_{0}$ is not available in the literature ${ }^{3}$. Therefore, we are unable to average $f\left(\mathbf{r}, \mathbf{r}^{*} \mid \varepsilon, \phi\right)$ over $\varepsilon$ to obtain $f\left(\mathbf{r}, \mathbf{r}^{*} \mid \phi\right)$. However, since the PDF of $\phi$ is known, we may consider the maximum a posteriori (MAP) approach to detect $\phi_{0}$. From the Bayesian's rule,

$$
\begin{gathered}
f\left(\phi \mid \mathbf{r}, \mathbf{r}^{*}, \hat{\varepsilon}_{0}\right)=\frac{f\left(\mathbf{r}, \mathbf{r}^{*}, \hat{\varepsilon}_{0}, \phi\right)}{f\left(\mathbf{r}, \mathbf{r}^{*}, \hat{\varepsilon}_{0}\right)}=\frac{f\left(\mathbf{r}, \mathbf{r}^{*} \mid \hat{\varepsilon}_{0}, \phi\right) f\left(\hat{\varepsilon}_{0}, \phi\right)}{f\left(\mathbf{r}, \mathbf{r}^{*} \mid \hat{\varepsilon}_{0}\right) f\left(\hat{\varepsilon}_{0}\right)} \\
=\frac{f\left(\mathbf{r}, \mathbf{r}^{*} \mid \hat{\varepsilon}_{0}, \phi\right) f\left(\hat{\varepsilon}_{0}\right) f(\phi)}{f\left(\mathbf{r}, \mathbf{r}^{*} \mid \hat{\varepsilon}_{0}\right) f\left(\hat{\varepsilon}_{0}\right)}=\frac{f\left(\mathbf{r}, \mathbf{r}^{*} \mid \hat{\varepsilon}_{0}, \phi\right)}{\pi f\left(\mathbf{r}, \mathbf{r}^{*} \mid \hat{\varepsilon}_{0}\right)} .
\end{gathered}
$$

Note that the denominator is independent from $\phi$. Then $\phi_{0}$ is estimated from

$$
\hat{\phi}_{0}=\arg \max _{\phi} f\left(\mathbf{r}, \mathbf{r}^{*} \mid \hat{\varepsilon}_{0}, \phi\right),
$$

This gives exactly the same result as (32).

\footnotetext{
${ }^{3}$ If CFO is assumed to be uniformly distributed in $[-0.5,0.5]$, then similar process can be conducted.
}

\section{Discussions}

Since MLE3 includes the complicated Bessel function, we will mainly provide the discussions on MLE1. As will be seen in the later simulations, all these three estimators perform identically within the regular SNR region.

1) Circular Transmissions: The ML CFO estimation for circular transmissions can be obtained by simply setting the elliptic variance of $s_{i}$ as zero. In this case, $\mathbf{B}$ and $\mathbf{U}$ become zero matrices. Hence, the PO cannot be estimated. However, the CFO could still be estimated from

$$
\begin{aligned}
\hat{\varepsilon}_{0}=\arg \max _{\varepsilon}-\mathbf{r}^{H} \mathbf{Y r} & \\
=\arg \max _{\varepsilon}{ }_{i=0}^{\mu-1} \Re\left(r_{i}^{*} r_{2 \mu-i-1}\right. & \left.+r_{i+N}^{*} r_{N+2 \mu-i-1}\right) \\
& \quad \times e^{j 2 \pi(2 i-2 \mu+1) \varepsilon}
\end{aligned}
$$

Note that, $\mu>0$ is required, otherwise $-\mathbf{r}^{H} \mathbf{Y r}$ is independent of $\varepsilon$, as explained previously. The estimator (44) can be easily understood in the noise free environment, in which case each term in (44) achieves its maximum at $\varepsilon=\varepsilon_{0}$. For example, $r_{u}=r_{u-1} e^{j 2 \pi \varepsilon_{0}}$ holds if there is no noise, and clearly, the term $\Re\left\{r_{u-1}^{*} r_{u} e^{-j 2 \pi \varepsilon}\right\}$ in (44) achieves its maximum at $\varepsilon=\varepsilon_{0}$. Estimator (44) could never provide the ML estimation for non-circular transmissions, and there is considerable performance loss if one still uses (44) for noncircular transmissions, as will be shown in the simulations later.

2) $C F O$ Tracking: Tracking the residue $\mathrm{CFO}$ and $\mathrm{PO}$ is also possible by considering the block with index $m>0$. Namely, for each new received block with $m>0$, we can estimate new $\mathrm{CFO}$ and PO corresponding to this specific block. If the $m$ th OFDM block is considered, we may regard $2 \pi m(N+$ $2 \mu) \varepsilon_{0}+\phi_{0}$ as the equivalent PO and carry on the similar steps to estimate CFO and PO. The PO estimator (32) could then be rewritten as

$$
\begin{aligned}
\hat{\phi}_{0} & =\arg \max _{\phi} \Re\left\{e^{-j 2(2 \pi m(N+2 \mu) \varepsilon+\phi)} \mathbf{r}^{T} \mathbf{U r}\right\} \\
& =\frac{1}{2} \angle e^{-j 4 \pi m(N+2 \mu) \varepsilon} \mathbf{r}^{T} \mathbf{U r} .
\end{aligned}
$$

Nevertheless, the ML CFO estimator (33) remains unchanged.

\section{Simulation Results}

In this section, we examine the proposed estimators under various scenarios. We consider OFDM systems with $N=64$ subcarriers (consistent with IEEE 802.11a standard [4]). In each example, $M_{q}=500$ Monte Carlo runs are conducted to average the simulation results. All symbols are assumed to be obtained from a BPSK constellation, namely, $E_{s}=E_{c}$. The normalized mean square errors is defined as

$$
\begin{aligned}
& \operatorname{NMSE}(\varepsilon)={\frac{1}{M_{q}}}_{i=1}^{M_{q}} \frac{\left|\hat{\varepsilon}_{0, i}-\varepsilon_{0}\right|^{2}}{\omega^{2}}, \\
& \operatorname{NMSE}(\phi)=\frac{1}{M_{q}}{ }_{i=1}^{M_{q}} \frac{\left|\hat{\phi}_{0, i}-\phi_{0}\right|^{2}}{\pi^{2}},
\end{aligned}
$$




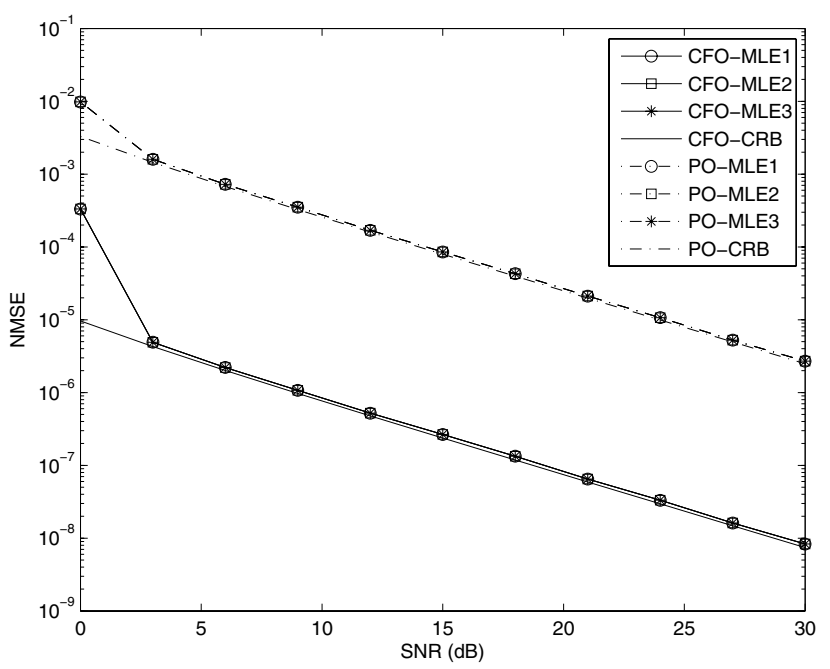

Fig. 1. Performance comparison between the MLE1, MLE2, and MLE3 in DCT-OFDM.

where $\omega=1 / N$ if $\varepsilon_{0}<1 / N$ and $\omega=\varepsilon_{0}$ otherwise. The parameters $\hat{\varepsilon}_{0, i}$ and $\hat{\phi}_{0, i}$ are the estimates of $\varepsilon_{0}$ and $\phi_{0}$ in the $i$ th Monte Carlo run.

\section{A. DCT-OFDM}

The normalized $\mathrm{CFO}$ is chosen as large as $\varepsilon_{0}=0.2$ (more than 12 subcarrier spacings) and PO is set as $\pi / 3$. We first compare our three estimators when both the prefix and suffix has length 8. The CFO and PO NMSEs versus SNR performances are shown in Fig. 1. The Cramer-Rao bounds (CRBs) [16] for CFO and PO estimations are displayed as well. From the figure, it can be seen that all three estimators provide the same performance. Moreover, they agree with the $\mathrm{CRB}$ very well, which is consistent with the general belief that the ML estimation can reach the CRBs asymptotically.

Next we examine how the performance differs by using different length of the prefix and the suffix. From the previous example, we know that all three proposed estimators perform identically. Hence, we will only present the result from MLE1 for the conciseness of the figure. The CFO and PO NMSEs versus SNR performances, for $\mu=0,2,4,8$ are drawn in Fig. 2 with their corresponding CRBs. From the figure, we can find that the CFO estimation accuracy is improved when $\mu$ is increased, say $4 \mathrm{~dB}$ when $\mu$ is increased from 0 to 8 . However, the PO estimation accuracy does not change much by varying the value of $\mu$.

We then illustrate the performance of the estimator (44) under the considered BPSK transmissions. Since $\mu$ for (44) must be greater than zero, we will select three values as $\mu=$ 2, 4, 8. Fig. 3 shows CFO NMSEs versus SNR performance for both the estimator (44) and the ML estimator (33). It can be seen that the performance loss is significant if one ignore the non-circularity of the transmitted symbols and apply the circular estimator (44) directly. Especially when $\mu$ is small, the performance loss is amazingly large. Therefore, DCT-OFDM

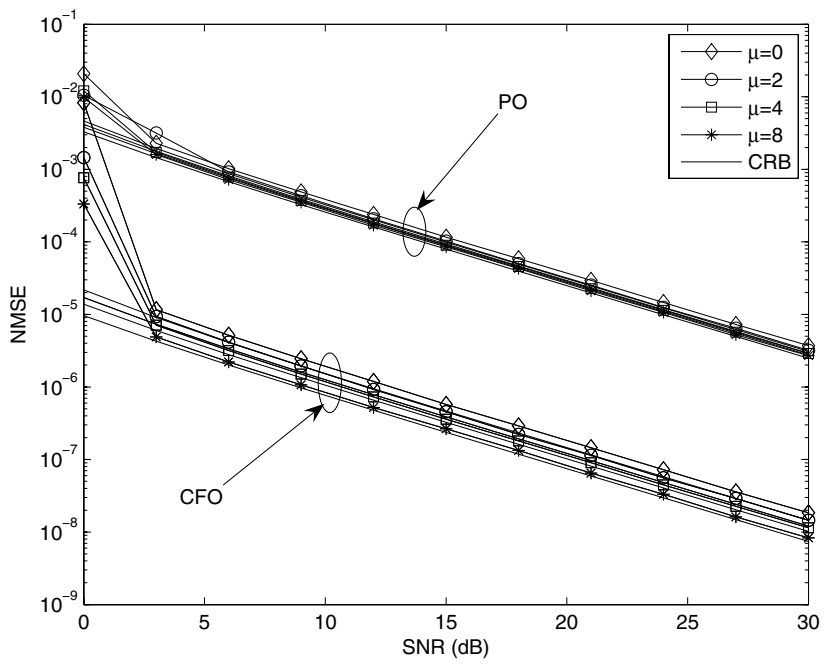

Fig. 2. Performance comparison of the CFO/PO estimations for different $\mu$ in DCT-OFDM.

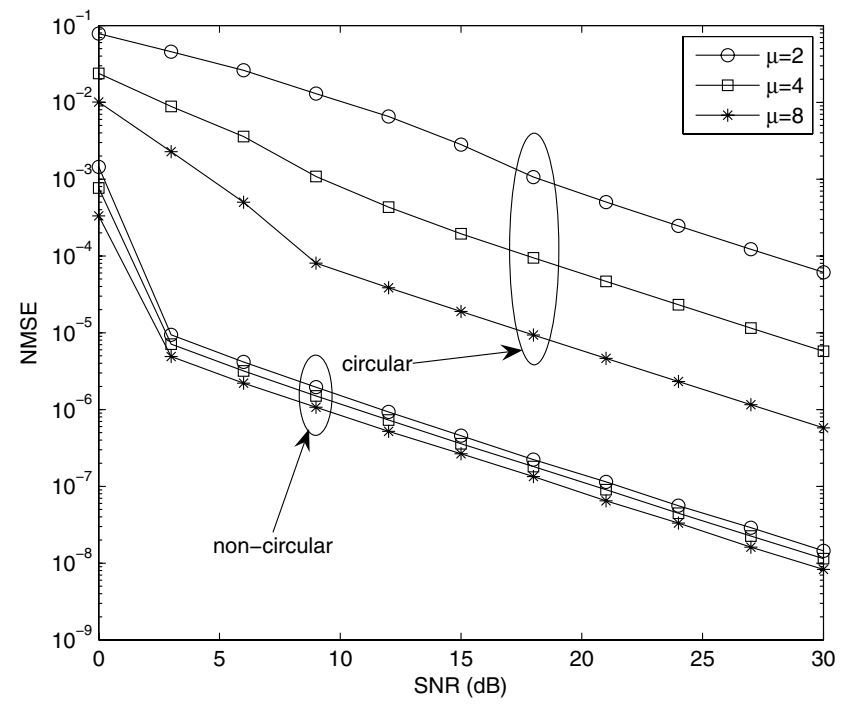

Fig. 3. Comparing CFO estimation of circular and non-circular estimators in DCT OFDM system.

is sensitive to whether the transmission is circular or noncircular, and ignoring the non-circularity, if any, will greatly drop the performance of DCT OFDM systems.

\section{B. Comparison Between DCT-OFDM and DFT-OFDM}

One may be curious that how the performance of these two OFDM systems differs for the non-circular transmission considered. We will study this issue in this example. Since DFT-OFDM is also considered, the parameters change to $\varepsilon_{0}=0.15 / N$, and $\phi_{0}=\pi / 3$. For a fair comparison, the total amount of redundancy added to one OFDM block is made the same, namely, $L=2 \mu$. The CFO and PO NMSEs versus SNR performances are shown in Fig. 4 and Fig. 5, respectively. In 


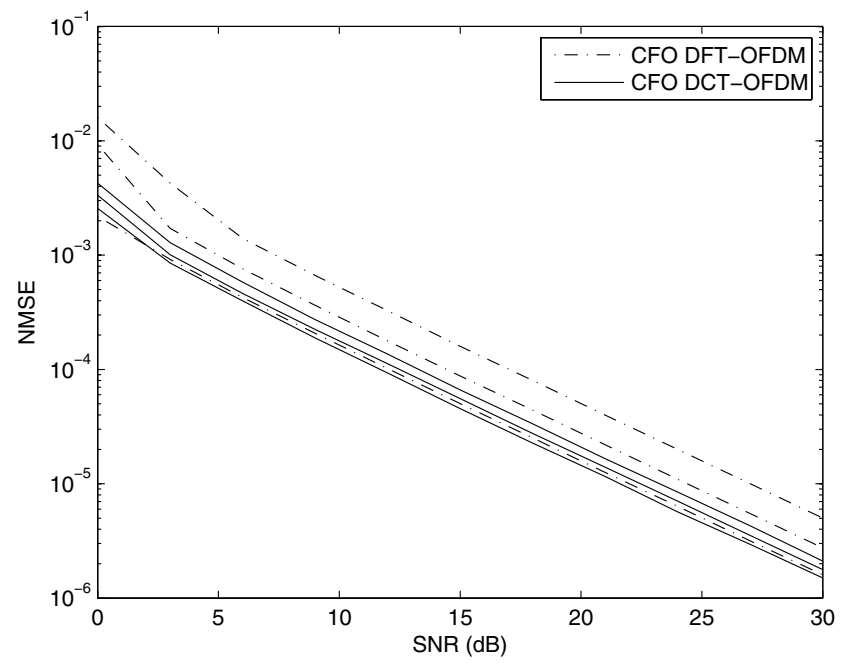

Fig. 4. Comparison between DCT-OFDM and DFT-OFDM under noncircular transmission, CFO estimation.

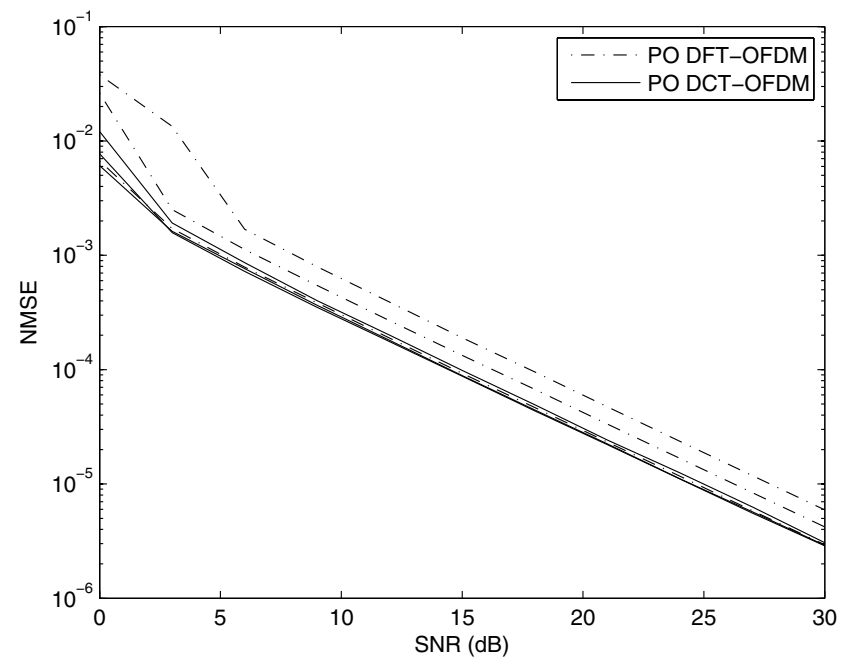

Fig. 5. Comparison between DCT-OFDM and DFT-OFDM under noncircular transmission, $\mathrm{PO}$ estimation.

each figure, the solid lines from up to down represent the DCT-OFDM with $\mu=2,4,8$, whereas the dashed lines from up to down represent the DFT-OFDM with $L=4,8,16$. Two phenomena are observed. 1) The DCT OFDM could provide better performance than the DFT OFDM, especially when the amount of the redundancy added to one OFDM block is small. 2) The performance of the DCT-OFDM vary slightly when we change the value of the $\mu$. However, the performance of the DFT-OFDM is affected greatly when we change $L$. As a result, the estimators in DCT-OFDM is more robust to the amount of the redundancy than those in DFT-OFDM. Clearly, both these two phenomena suggest extra advantages of the DCT-OFDM over the DFT OFDM.

\section{CONCLusions}

In this paper, we considered the non-circular transmissions and proposed three ML joint $\mathrm{CFO}$ and PO estimators for the newly raised DCT-OFDM systems. In addition to those mentioned in [5], [6], we found new advantages of the DCTOFDM over the DFT-OFDM. First of all, if DCT based OFDM is applied, the CFO estimation range could be increased from only one subcarrier spacing to its maximum. Secondly, the $\mathrm{CFO}$ and PO estimation in DCT-OFDM is more accurate than those in DFT-OFDM. Lastly, the CFO estimation in DCT OFDM is robust to the amount of the redundancy inserted than in DFT-OFDM. Simulation results clearly support our analysis and discussions.

\section{REFERENCES}

[1] "Radio broadcasing system, digital audio broadcasting (DAB) to mobile, portable, and fixed receiver," Eur. Telecommun. Stand. Inst., SophiaAntipolis, Valbonne, France, ETS 300 401, 1995-1997.

[2] "Digital broadcasting system television, sound, and data services; framing structure, channel coding, and modulation digital terrestrial television," Eur. Telecommun. Stand. Inst., Sophia-Antipolis, Valbonne, France, ETS 300 744, 1996.

[3] "Broadband radio access networks (BRAN): high performance radio local area networks (HIPERLAN), type 2; Systems overview," Eur. Telecommun. Stand. Inst., Sophia-Antipolis, Valbonne, France, ETR 101 $683114,1999$.

[4] "Wireless LAN medium access control (MAC) and physical layer (PHY) specifications: high speed physical layer in the 5 GHZ band," IEEE802.11a, 1999

[5] N. Al-Dhahir, H. Minn, and S. Satish, "Optimum DCT-based multicarrier transceivers for frequency-selective channels," IEEE Trans. Commun., vol. 54, pp. 911-921, May 2006.

[6] P. Tan, and N.C. Beaulieu, "Precise bit error probability analysis of DCT OFDM in the presence of carrier frequency offset on AWGN channels," in Proc. IEEE GLOBECOM'05, vol.3, pp. 1429-1434, St.Louis, Missouri, USA, Nov. 2005

[7] T. Pollet, M. van Bladel, and M. Moeneclaey, "BER sensitivity of OFDM systems to carrier frequency offset and Wiener phase noise," IEEE Trans. Commun., vol. 43, pp. 191-193, Feb. 1995.

[8] J.J. van de Beek, M. Sandell, and P.O. Borjesson, "ML estimation of time and frequency offset in OFDM systems," IEEE Trans. Signal Processing, vol. 45, pp. 1800-1805, July, 1997.

[9] J. Galy, and C. Adnet, "Blind separation of non-circular sources," Proc. IEEE SSAP'00, Pennsylvania, USA, pp 315-318, Aug. 2000.

[10] T. Fusco, and M. Tanda, "Blind symbol timing, frequency offset and carrier phase estimation in OFDM systems," in Proc. 37th Asilomar Conf., Pacific Grove, USA, vol. 1, pp. 179 - 183 Nov. 2003.

[11] I.A. Tasadduq, and R.K. Rao, "OFDM-CPM signals," IEEE Electro. lett. vol. 38, pp. 80-81, Jan, 2002

[12] E.Y. Lam, and J.W. Goodman, "A mathematical analysis of the DCT coefficient distributions for images," IEEE Trans. Image Processing, vol. 9 , pp. 1661-1666, Oct. 2000.

[13] B. Picinbono, "Second order complex random vectors and normal distributions," IEEE Trans. Signal Processing, vol. 44, pp. 2637-2640, October, 1996.

[14] T. Cui, and C. Tellambura, "Power delay profile and noise variance estimation for OFDM," IEEE Commun. Lett., vol. 10, pp. 25-27, Jan. 2006.

[15] H.L. van Tree, "Detection, estimation, and modulation theory," Weiley Interscience, 2001.

[16] J-P. Delmas, and Habti Abeida, "Stochastic cramer-rao bound for noncircular signals with application to DOA estimation," IEEE Trans. Signal Processing, vol. 52, pp. 3192-3199, Nov. 2004. 